\title{
Escenarios y retos de la televisión pública en España
}

Juan Luis Manfredi Sánchez

IE University

\section{Palabras clave}

Television pública, servicio público, Europa, España, estrategia, digital

\section{Resumen}

La televisión pública en España se enfrenta al reto de adaptarse y adecuarse a la expansión y la consolidación de los medios digitales globales. No se han acordado soluciones estables a las contradicciones del control editorial, la gestión económica, los contenidos y el buen gobierno corporativo, lo que dificulta la determinación de la misión de la televisión pública y las estrategias a seguir.

La modernización del ordenamiento legal, la autonomía de los servicios informativos y la gestión de los canales digitales son avances positivos. No obstante, son continuistas, porque no parecen ligados de un proyecto común, con vocación de estabilidad. La financiación sigue siendo un asunto espinoso. La libertad que promueve la Comisión Europea en la definición del servicio público audiovisual degenera, en el caso español, en la indefinición sobre los contenidos y los medios de pago. La nueva autoridad audiovisual está llamada a liderar estas cuestiones y establecer parámetros homogéneos con el entorno europeo.

Ha comenzado un nuevo proyecto que tendrá que establecer las bases de la misión, la dinámica y el alcance de RTVE y, por extensión, de los operadores autonómicos. Ha llegado el momento de pensar hacia dónde queremos encaminar la televisión pública en los próximos diez años. 


\title{
Scenarios and challenges of public television in Spain
}

\section{Keywords}

Public television, public service, Europe, Spain, strategy, digital

\begin{abstract}
Spanish Public Service Television faces the challenge to adapt and to adjust to the current expansion and consolidation of the global digital media. At the moment, RTVE lacks of stable solutions to the contradictions of editorial control, financial management, content and corporate governance, which makes difficult to establish the vision, the mission and the strategies to follow up.

The modernization of the legal system, the autonomy of the newsroom and the excellent management of the digital channels are positive developments. However, the main policies maintain the given situation before these positive changes. Why? They do not appear linked in a common. Funding remains a thorny issue. The freedom that promotes the European Commission in the definition of public service broadcasting degenerates, in the Spanish case, in the uncertainty about the content and means of payment. The new audiovisual authority is called to lead these issues and establish parameters homogeneous in the European environment.

The Spanish Government starts a new project that will have to lay the groundwork for the mission, the dynamics and scope of RTVE and, by extension, regional operators. Now is the time to think about where we want to steer public television in the next ten years.
\end{abstract}

\section{Financiación}

El artículo se enmarca dentro del Proyecto de Investigación I+D+I "Impacto de Internet en la redefinición del servicio público de las televisiones públicas autonómicas españolas" (Referencia del Proyecto: CSO2009-11250/SOCI), aprobado por el Ministerio de Ciencia y Tecnología para el periodo 2009-2012. IP: Juan Carlos de Miguel Bustos.

\section{Autor}

Juan Luis Manfredi [Juan.manfredi@ie.edu.] es periodista y profesor de IE University. 


\section{La nueva etapa del sistema audiovisual: la expansión de los medios digi- tales globales}

La televisión pública ha modelado el sistema de comunicación de los países europeos desde su aparición, en la medida en que los estados han definido el marco legal, los límites de la libertad de empresa y el monopolio, las telecomunicaciones y los derechos individuales (Murciano, 2006). Los países democráticos se decantaron por una televisión monopolística y vinculada al sistema político a través de diferentes resortes (canon, dirección general o censura), mientras que las dictaduras mediterráneas y de Europa del Este insertaron la televisión directamente dentro de los órganos de gobierno y la burocracia (Manfredi, 2004: 275). En ambos casos, la intervención en el mercado tenía como objetivo el acceso universal (función política), el establecimiento de unos estándares de ocio y de entretenimiento (función cultural) y el liderazgo de la industria audiovisual y tecnológica (función económica). El método de financiación más habitual fue el establecimiento de canon o una cuota por el acceso universal a los medios audiovisuales, facilitando la planificación estratégica y financiera de los operadores.

El sistema de comunicación, durante el siglo XX, puede dividirse en cuatro grandes etapas, de acuerdo con la posición de la televisión pública en el mismo (Picard, 2001: 67). La primera, anterior a 1957, es la era de la imprenta y el servicio universal de radio. La segunda, entre 1957 y 1970, sienta las bases del servicio público de televisión y consolida la radio como el medio de comunicación más popular. En este periodo, se fundan la mayoría de las televisiones públicas y se dan las características socioeconómicas para su expansión en los hogares. Entre 1970 y 1985, el mercado audiovisual comienza un gradual proceso de liberalización y de desregulación. En el seno de la Comunidad Económica Europea, el Tribunal de Luxemburgo reconoce en la Sentencia Sacchi que la televisión es una actividad económica (“asimilada”). El mantenimiento del monopolio es válido para los objetivos específicos de la misión de servicio público, pero el resto de la actividad debe adecuarse a la libre prestación de servicios. Este enfoque liberalizador terminará en la creación de una política audiovisual europea (Humphreys, 1996:261) y, en particular, en la Directiva 89/552/CEE, conocida como la Directiva Televisión Sin Fronteras. La cuarta etapa, fechada en 1985 y vigente hasta la actualidad, es la era de los medios como negocio global, que incluye el auge de la publicidad transnacional, la liberalización de los servicios, la consolidación de los conglomerados empresariales mixtos y el declive de la televisión pública.

Como consecuencia de estas medidas políticas y de la globalización de los patrones comerciales de ocio y consumo, se ha creado un mapa audiovisual complejo, que ha fragmentado las audiencias y ha roto definitivamente el monopolio en el ámbito de la distribución y la programación, la producción y la emisión. La digitalización de los procesos, la atomización de las audiencias, la multiplicación de la oferta de libre acceso mediante la TDT y la consolidación de Internet y los 
nuevos medios como dispositivos de consumo conducen hacia una nueva etapa en la dinámica y el alcance de la televisión pública.

La nueva etapa se enfrenta a un escenario novedoso en la gestión y la administración de los negocios audiovisuales. La cadena de valor tradicional, basada en el empaquetamiento de contenidos (propios o ajenos) y su emisión dentro de una parrilla cerrada está obsoleta. El entorno limitado de la empresa audiovisual se ha visto desbordado por la desregulación y la liberalización, el cambio en la estructura de la industria de la comunicación, el exceso de capacidad de muchos subsectores, la proliferación de fusiones, adquisiciones y alianzas, la preocupación por temas medioambientales, el menor proteccionismo, los cambios en las expectativas de los consumidores, la disrupción tecnológica, la emergencia de bloques comerciales y la competencia global (Caro, 2006: 74-82). El cambio es progresivo, pero imparable (Pew, 2010: 28 y Academia de TV, 2010: 12-13).

Por otro lado, los nuevos retos se plantean con numerosas cuestiones pendientes tales como la crisis de legitimidad y de identidad de los operadores públicos (Richeri, 1994). La legitimidad se plantea en la medida en que, roto el monopolio, la televisión pública tiene que ofrecer unos contenidos diferentes y de valor para la audiencia sin distorsionar las reglas del mercado. Sin la racionalidad del espectro y la publicidad limitadas, la identidad inicial marcada por el patrón de la BBC está en cuestión. ¿Qué contenidos pueden crear valor para el espectador y generar ventajas competitivas en el mercado sin desfigurarlo? Los servicios informativos cumplen estas expectativas.

El profesor Sánchez-Tabernero añade tres contradicciones específicas. A saber: el control editorial por parte de la esfera política, la gestión económica y financiera y los contenidos (Sánchez-Tabernero, 2005). Finalmente, habría que sumar la vaguedad de los principios de buen gobierno, transparencia y responsabilidad social, que son problemas comunes a los operadores privados (Arrese, 2006; Manfredi, 2009).

La falta de definición de estos problemas plantea un futuro incierto para el gobierno audiovisual y la misión de la televisión pública, especialmente en España donde la televisión se emplea con instrumento, no como medio para el desarrollo económico (industria de contenidos, I+D, tecnología, exportaciones) y social (diversidad y pluralismo). Sin un grado de consenso parlamentario en las cuestiones fundamentales, parece poco probable que los gestores de TVE puedan diseñar una estrategia sólida para competir en el mercado audiovisual en el siglo XXI.

\section{La lenta modernización de TVE}

El Estatuto de 1980 (Ley 4/1980, de 10 de enero) ha favorecido un tipo de televisión pública estática e inmovilista, dependiente de la esfera política para el ejercicio de sus funciones y de la publicidad para la toma de decisiones. La 
situación empeoró tras la aparición de las televisiones privadas (Ley 10/1988, de 3 mayo, de Televisión Privada, vigente hasta el 1 de mayo de 2010), lo que condujo a un déficit permanente y al cuestionamiento de la misión de TVE y las televisiones autonómicas en un mercado abierto a la competencia.

El marco legal era inadecuado e insuficiente para afrontar el cambio de modelo y presentar un proyecto viable a medio plazo. Se han tomado tres grandes medidas para afrontar el cambio con garantías y dar un salto de calidad en la legislación, la gestión y las decisiones internas.

\subsection{El nuevo ordenamiento legal}

En 2004, el gobierno socialista marca como prioridad la reforma de los medios de titularidad pública ante la pérdida de credibilidad y la pésima gestión económica y financiera. Se nombra un comité de expertos que elabora el "Informe para la reforma de los medios de comunicación de titularidad del Estado”, el primer documento de este orden en la España democrática. Sobre esta base se elaboran tres leyes que han modificado el orden jurídico.

La primera norma es la Ley 17/2006, de 5 de junio, de la radio y la televisión de titularidad estatal. Deroga el Estatuto, pero mantiene la idea de función de servicio público basado en la programación de calidad y en el fomento de la producción española y europea, así como el desarrollo de servicios conexos. El primer objetivo declarado es garantizar la imparcialidad y la independencia de los servicios informativos. Entre otras medidas, se crea el Consejo de Informativos como órgano de participación de los profesionales de la casa. La Ley crea la Corporación RTVE, de capital social estatal, y la disolución del anterior Ente público. Establece la creación de un mandato marco para el establecimiento de los objetivos de la función de servicio público. En cuanto a la financiación, se valida el modelo mixto, si bien se reduce la plantilla, el gasto corriente y la inversión.

Tres años más tarde, se aprueba la Ley 8/2009, de 28 de agosto, de financiación de la Corporación de Radio y Televisión Española. Se pretende que la nueva entidad se gestione adecuadamente, vinculando la independencia de los informativos y de los contenidos con la viabilidad económica y financiera. La norma suprime la publicidad retribuida, salvo en determinadas excepciones (artículos 7.3. y 7.5.), obligando a los operadores privados y a otros actores concesionarios a contribuir en el mantenimiento de la Corporación. Por su parte, los Presupuestos generales del Estado compensarán el cumplimiento de las obligaciones de servicio público referidas en el nuevo ordenamiento legal.

Por último, se ha aprobado la Ley 7/2010, de 31 de marzo, General de la Comunicación Audiovisual. Compendia la regulación de la comunicación audiovisual de cobertura estatal y establece las normas básicas para favorecer la libre competencia (disrupción tecnológica) y la protección de los derechos de los ciudadanos. Con esta meta, fija las obligaciones de los operadores en relación 
con el pluralismo, los derechos de los prestadores de servicio, los límites de la publicidad, las posibilidades de las nuevas tecnologías, la ordenación de las licencias y concesiones, la definición del servicio público de televisión (artículo 40), la exigencia de transparencia empresarial y la creación del Consejo Estatal de Medios Audiovisuales, entre otras cuestiones.

El compendio legal supera la dispersión legal que ha caracterizado el sector audiovisual y tecnológico, tratando de adecuarse a las nuevas necesidades del mercado y la demanda de los públicos. No obstante, tiene un marcado carácter continuista y termina de afrontar la dos cuestiones fundamentales en la definición de la misión de la televisión pública estatal. Son los siguientes: qué contenidos reales (de mercado) se ajustan a la función de servicio público en detrimento de maximizar la audiencia y quién o cómo se financian, dada la improbable creación de un canon, los límites que impone el Tribunal Justicia de la Unión Europea (TUE) y los límites impuestos por la Comisaría de Competencia. Esta indefinición genera incertidumbres.

\subsection{La gestión de los nuevos canales}

Desde el punto de vista de la gestión y la administración, la digitalización de los servicios de TVE ha multiplicado las ventanas, la notoriedad y el impacto en la opinión pública. De acuerdo con la matriz de Ansoff (1985), se ha redefinido el product-mix que la corporación puede producir y que los mercados demandan. Estas decisiones influyen en la gestión de los contenidos, su financiación, la relación con los públicos y el cumplimento con la función de servicio público. Se ha tratado de diversificar las fuentes, el entretenimiento y el ocio para captar nuevos públicos, rompiendo la tendencia homogeneizadora de los años ochenta y noventa (Manfredi, 2008:137).

En el espacio de la TDT, TVE ha optado por la especialización como ventaja competitiva. Mediante la agregación de contenidos, se han creado ofertas atractivas para mercados nicho que habían quedado fuera de la televisión digital gratuita. Esa estrategia, con el soporte y la estructura de RTVE, han reforzado la identidad de la cadena. Siguiendo a Porter (2000), TVE ha generado valor para los clientes (audiencia nicho) y ha reducido costes (aprovechamiento de los archivos y la compra en bloque de derechos). Junto con la oferta tradicional (La 1 y La 2), se han creado los siguientes canales: 24horas, Clan TV, Teledeporte, Cultural.es y Clásico. En términos de audiencia, Clan TV es un referente, ya que es la televisión temática con más audiencia (4,1\% en septiembre de 2010) en el mercado español, mientras que Teledeporte lidera el mercado de los temáticos deportivos (1,38\%). 24horas se mantiene en el 0,87\% de audiencia, ligeramente por encima de CNN Plus $(0,70 \%)$.

En Internet y los nuevos medios, TVE ha apostado por la adecuación de los contenidos de rtve.es a las nuevas pantallas. La estrategia desarrollada por Ro- 
salía Lloret (2008-2010) se ha basado en "hacer servicio público en todo lo que ofrecemos" (Varela, 2008), facilitando el acceso y llegando a todo tipo de audiencias. Dicha orientación hacia el espectador y el oyente se ha plasmado en el acceso a los programas, los videos informativos, la radio en directo, contenidos en podcast, noticias y deportes. También se han lanzado blogs de periodistas y se ha buscado una mayor participación de la audiencia en programas concretos.

El resultado ha sido excelente: la audiencia ha crecido el 121,21\% entre marzo de 2009 y abril de 2010. RTVE.es es el primer portal en español de un medio audiovisual con 7,5 millones de usuarios únicos y 230 millones de páginas vistas, según datos publicados por RTVE.es. Los contenidos de televisión son los más seguidos por encima de la radio o la zona infantil. Por otro lado, según OJD Interactiva, en agosto de 2010, RTVE.es lidera el segmento de las televisiones públicas y privadas con 6,4 millones de visitantes únicos, por encima de Antena 3(5,3 millones) y Telecinco (5,8 millones).

\subsection{Los códigos internos de la Corporación}

La Corporación ha facilitado la creación de dos instrumentos recogidos en la nueva legislación audiovisual para mejorar la función de servicio público. En el ámbito informativo, se ha aprobado el Estatuto de Información de RTVE es un documento interno por el que el sujeto se obliga a respetar las buenas prácticas y los códigos deontológicos, al tiempo que recoge los derechos de los profesionales. Tiene validez jurídica y sirve como órgano interno de participación. Se aprobó por referendo el 18 de abril de 2008. El Estatuto tiene asignadas las funciones de velar por la independencia de los profesionales, promover la independencia editorial, informar sobre la línea editorial y la programación informativa, la participación en los libros de estilo e informar sobre las propuestas de nombramientos. Dichas tareas están en sintonía con las obligaciones al uso (Aznar, 1999:43-45).

En el plano de los contenidos, el Consejo de Administración ha aprobado el "Código de Autorregulación para la defensa de los derechos de los menores" con fecha 21 de julio de 2010. Este instrumento tiene como meta proteger los contenidos que ven los menores en los medios y cómo éstos se ven reflejados en la programación audiovisual. En el mismo, se amplía la programación familiar durante los fines de semana y los festivos, así como las franjas de protección. Los contenidos se clasificarán de acuerdo con las recomendaciones de edad, según el comportamiento social, la violencia, la conflictividad y el sexo. La autorregulación consiste en el establecimiento de los límites por uno mismo sin intervención de terceros. Es un mecanismo de autocontrol, en la línea de "Código de Autorregulación de Contenidos Televisivos e Infancia", aprobado por TVE, Antena 3, Tele 5 y Canal Plus el 9 de diciembre de 2004 y firmado en colaboración con el Gobierno. 
En el primer caso, el Estatuto ha tenido un bajo impacto en la redacción y en los nombramientos, dos de las principales tareas. En la misma línea, el nuevo código de autorregulación está por ver. La experiencia de 2004 ha resultado pobre y poco efectiva (García, 2006 y Agejas, 2007), porque las televisiones privadas han apostado por maximizar las audiencias mediante la rentabilización económica de los contenidos. TVE y las televisiones autonómicas no han sabido desarrollar unos contenidos exclusivos que faciliten una estrategia diferenciadora (Porter, 1996), por lo que han caído en una programación homogeneizadora (Fernández y Santana, 2000: 372; Artero, 2008: 279).

\section{Retos pendientes}

El proceso de modernización de TVE es un hecho visible. Se ha mejorado el marco legal, la gestión de los nuevos canales y se ha dotado de más autonomía a los servicios informativos. Estos avances son positivos, pero quedan todavía numerosas cuestiones pendientes que apuntan, de momento, a un modelo continuista antes que innovador en la manera de administrar la televisión pública digital. Todavía faltan instrumentos legales y hábitos políticos para consolidar el proyecto y adecuarlo a los estándares europeos de referencia.

El primer reto es la consolidación del marco legal y la estabilidad del gobierno audiovisual. España se ha caracterizado por los vaivenes en la industria, lo que ha perjudicado seriamente la creación de empleo, las inversiones y el desarrollo de talento. La Ley 7/2010, de 1 de abril, nace con vocación de permanencia, a pesar de de los votos en contra del PP y del PNV, así como las críticas de IU. Es necesario que se mantenga la legislación audiovisual y se incremente el consenso parlamentario en el Estado y en las autonomías para favorecer dicho sector. Todavía se puede mejorar: está pendiente la adecuación de las televisiones autonómicas a la nueva legislación y el desarrollo reglamentario del Consejo Audiovisual estatal. Las deficiencias de la ley pueden cubrirse con sucesivas mejoras, pero no podemos permitirnos volver al caos legislativo de la década de los noventa y la primera del siglo XXI.

Para afrontar este desafío, el gobierno tiene que impulsar el consenso con el principal partido de la oposición y generar un debate continuado dentro de las directrices marcadas por la Unión Europea y el Consejo de Europa. Esta decisión implica la responsabilidad de la esfera política, así como el incremento de la participación de los actores de la industria audiovisual. Los foros de debate, los libros blancos y la formación continuada son pasos dentro de una hoja de ruta que reúna los intereses de los creadores de contenidos, los intereses empresariales y económicos y los analistas de la industria. La mejora de la calidad legislativa afecta a todos los actores de la industria y exige un seguimiento permanente, vistas la velocidad de los cambios y las consecuencias socioeconómicas. 
El segundo desafío es la financiación, porque el actual modelo no está cerrado. En efecto, en el entorno europeo, se admite la financiación de los medios públicos si bien obliga a los estados a "asegurar el control periódico y efectivo del uso del dinero público para evitar la sobrecompensación y el subsidio cruzado, así como para evaluar el nivel y el uso de las reservas de servicio público" (European Commission, 2009:18). La doctrina del Tribunal de Justicia avala esta posición cuando establece cuatro requisitos para la compensación económica procedente del dinero público y para evitar el falseo de la competencia (Caso Almark, C-280/00). Las condiciones que marca dispone que la empresa deben ser responsable de la ejecución del servicio público, que los parámetros de compensación deben ser transparentes y objetivos, que la compensación debe ser proporcionada y que, en caso de ausencia de contratación pública, la compensación se ajustará al análisis de costes de mercado.

En la práctica, significa que "cada estado miembro es libre de elegir el significado de la financiación del servicio público audiovisual” (Bron, 2010:7) una vez que define dos variables: qué contenidos y cómo se paga. Si no se acotan ambas condiciones, el país miembro corre el riesgo de no superar los tests de eficiencia de los servicios públicos según las condiciones expuestas. La devolución de compensaciones por parte de TV2/Danmark (84,4 millones de euros) y Nos Netherland Broadcasting Corporation (76,3 millones de euros) expone la tenacidad de la Comisión Europea en este sentido.

La implantación de la transparencia empresarial (artículo 42.2 de la Ley 7/2010, de 1 de abril) y la contabilidad analítica (artículo 43.5 de la Ley 7/2010, de 1 de abril) son herramientas conducentes al cumplimiento de dichas obligaciones. Está pendiente la estabilidad financiera que asegure la viabilidad del proyecto. En relación con los contenidos el artículo 40 de la Ley $7 / 2010$, de 1 de abril, define el servicio público como un "servicio esencial de interés económico general que tiene como misión difundir contenidos que fomenten los principios y los valores constitucionales, contribuir a la formación de una opinión pública plural, dar a conocer la diversidad cultural y lingüística de España, y difundir el conocimiento y las artes, con especial incidencia en el fomento de una cultura audiovisual. Asimismo, los prestadores de servicio público de comunicación audiovisual atenderán a aquellos ciudadanos y grupos sociales que no son destinatarios de la programación mayoritaria”. Expresamente se prohíbe la cesión a terceros de la producción y la edición de los servicios informativos y de otros programas expresamente recogidos en el mandato marco. La definición de servicio es abierta, lo que facilita las iniciativas de todo tipo, sean éstas de interés público o no. Igualmente, corre el riesgo de competir en aquellas actividades de interés económico que tendrían que ser emitidas por las operadoras privadas, como por ejemplo el deporte profesional o los estrenos de cine no participados vía financiación, programa Media o similar.

De acuerdo con lo establecido en los artículos 2 y 3 de la Ley 17/2006, de 5 de junio, y el artículo 9 de la Ley 8/2009, de 28 de agosto, los contenidos de 
servicio público de RTVE cuentan con la ayuda pública directa y con tres tasas indirectas. Las televisiones privadas en abierto abonarán el 3\% de los ingresos; las televisiones de pago, el 1,5\%; y las operadoras de telecomunicaciones, el 0,9\% con el objetivo de compensar la salida de TVE del mercado publicitario, valorada en alrededor de 500 millones de euros. Las tasas han sido recurridas ante la Comisión Europea, que ha iniciado un procedimiento de infracción contra España por la tasas sobre los ingresos de las empresas de telecomunicaciones. Tal situación conduce a la no recaudación de alrededor de 250 millones de euros. Si se mantiene la escasez, la corporación volvería a entrar en déficit permanente y posible quiebra. Igualmente, la Comisión Nacional de la Competencia ha emitido un informe en el que señala que las tasas sobre los operadores puede ser un elemento de distorsión en la competencia generando asimetrías en los mercados afectados.

El tercer reto está vinculado al corazón de la televisión. El marco legal y la financiación mixta establecen la necesidad de asegurar la función de servicio público, lo que necesariamente conduce a la evaluación de los contenidos emitidos bajo la etiqueta de servicio público. En la cultura mediática europea está establecida la evaluación del servicio público por órganos competentes en la materia, que establecen los parámetros básicos para la programación, la financiación y la gestión de derechos audiovisuales.

La nueva Ley Audiovisual establece que la autoridad audiovisual evaluará los contenidos para saber si se ajustan a la misión de servicio público y si alteran la competencia en la industria audiovisual (artículo 41.3). Existen numerosos ejemplos de control exante o expost en la Europa mediática (Bron, 2010:18-25). En el Reino Unido, BBC Trust (homologable al Consejo de Administración) somete al "public value test" los contenidos de la emisora para evaluar el interés público de los mismos. Con esta herramienta, se conoce el coste, el impacto en la sociedad, la novedad y la duración del servicio. En Alemania, los operadores públicos registran sus necesidades económicas ante una comisión que evalúa las necesidades y sugiere ahorros posibles exante. A partir de este documento se establece el canon de cada Länder. En relación con los contenidos, éstos pasan un examen de tres pasos: hasta qué punto los contenidos alimentan las necesidades de la democracia, hasta qué punto contribuye o distorsiona la competencia y cuánto cuesta el servicio. En Irlanda, el control a posteriori se basa en el informe remitido al Ministerio de Comunicaciones, que evalúa el gasto público de la RTÉ y recomienda cómo revisar el canon.

Todo apunta a que estas y otras funciones recaerán en el Consejo Estatal de Medios Audiovisuales (artículos 44-54 de Ley 7/2010, de 1 de abril), que es el cuarto asunto pendiente de resolver. Por un lado, se trata de un organismo que existe en casi todos los países de la Unión Europea y del Consejo de Europa. Su misión consiste en velar y garantizar el ejercicio de la comunicación audiovisual, defender los derechos y las obligaciones establecidas por ley, apoyar la transparencia y el pluralismo del sector y, por último, favorecer la independencia y 
la imparcialidad de los medios públicos audiovisuales, en calidad de autoridad independiente supervisora y reguladora de la actividad de los medios de titularidad del Estado.

El riesgo de creación de un nuevo organismo público es la descoordinación con aquellos preexistentes, sobre todo con la Comisión del Mercado de las Telecomunicaciones (en relación con la convergencia) y con el Defensor del Pueblo (por los asuntos relacionados con el menor). Después de años de desgobierno audiovisual, el Consejo tiene que diseñarse muy delicadamente para evitar la duplicación de costes y de funciones en detrimento del buen funcionamiento del mercado y la industria. Igualmente, en tiempos de estrechez presupuestaria, la fusión con la Comisión podría ahorrar hasta 34 millones de euros. En nuestra opinión, el Consejo nace viciado al separarse de la Comisión.

\section{Un nuevo rumbo}

Estamos ante una nueva etapa en la historia de los medios públicos audiovisuales. TVE ha afrontado el reto con una modernización lenta, continuista y discutible, si bien por fin ha abandonado el sistema mediático creado en 1980. El proceso no ha terminado, sino que se encuentra en un momento fundamental para sentar las bases de la misión, la dinámica y el alcance de TVE y, por extensión, de las televisiones autonómicas.

En el ámbito empresarial, la estrategia tiene que reforzar la creación y el sostenimiento de la industria audiovisual española mediante la demanda de contenidos y de servicios de valor añadido. La Ley General de la Comunicación Audiovisual establece los límites a la externalización (artículo 40.1), que parecen favorecer la creación de un sector audiovisual independiente, el ahorro de costes fijos y la concentración en los servicios esenciales, sobre todo los servicios informativos. El riesgo de la externalización es la generación de un mercado de influencia dominante y la dependencia financiera de las pequeñas empresas. Los gestores de TVE tienen que velar por la competitividad y la creación de empleo a largo plazo, declinando las ofertas baratas y de consumo efímero. Tampoco parece sostenible el actual ritmo de crecimiento expansivo sin la aprobación previa de un contrato programa, un documento de valor (public value test o similar) y otras herramientas que delimiten las funciones y las ventanas.

La estrategia digital tiene que dar un salto de calidad, de acuerdo con los nuevos patrones de uso y consumo en los dispositivos móviles. Esto implica el desarrollo de nuevos productos digitales y el apoyo a la industria, como motor y soporte de productoras y creadores mediante la externalización (apoyo a la industria local) y mediante la creación de contenidos propios (uso de recursos internos, capacitación de empleados, liderazgo en competencias esenciales). Después del crecimiento de audiencia del periodo 2008-2010, toca favorecer el talento y el $\mathrm{I}+\mathrm{D}+\mathrm{i}$. 
En relación con la calidad y los estándares profesionales, TVE tiene que emplear las herramientas de las que se ha dotado para la gestión y liderar una programación de calidad. Los servicios informativos son una piedra de toque para medir el grado de cumplimiento de los criterios objetivos (rigor, profundidad, comprensión del mensaje, uso del lenguaje, diversidad de fuentes, realización o documentación) y los subjetivos (veracidad, respeto). La audimetría de rentabilidad social es un instrumento que tendrá que ser elaborado por TVE con el objeto de medir el impacto de los contenidos audiovisuales en la audiencia. No basta con medir GRP ni empaquetar audiencias, sobre todo cuando se ha acabado la etapa de acumular audiencias para ser vendida a los anunciantes y las agencias. La medición tiene que incluir nuevos parámetros objetivos (calidad y estándares profesionales) y subjetivos (satisfacción de la audiencia, impacto en la esfera democracia).

Por último, en el ámbito de la financiación, no cabe de momento el planteamiento del pago de un canon para el mantenimiento ciudadano de la televisión. Tampoco parece probable la vuelta a la publicidad convencional, porque las televisiones privadas han sido las principales beneficiarias y se opondrían sistemáticamente. Tienen argumentos: según los resultados publicados, han captado entre el 20 y el 30\% más de recursos, mejorando los resultados parciales frente al declive general de la inversión publicitaria. Ante esta tesitura, urge la definición de un modelo viable de financiación que concuerde con las directrices europeas y se ajuste a la legalidad española.

\section{Referencias}

Academia de Televisión (2010). La industria audiovisual en España. Escenarios de un futuro digital. Madrid: Fundación EOI.

Agejas Esteban, José Ángel (2007). La autorregulación televisiva: ¿responsabilidad o utopía?. En: Fundación COSO. La ética y el derecho de la información en los tiempos del postperiodismo. Valencia: Fundación COSO.

Ansoff, Igor (1985). Corporate Strategy. London: Penguin.

Arrese, Ángel (2006). Reflexiones sobre el buen gobierno corporativo en las empresas periodísticas. En: Doxa Comunicación, 4, 59-82.

Artero Muñoz, Juan Pablo (2008). El mercado de la televisión en España: oligopolio. Barcelona: Deusto.

Aznar, Hugo (1999). Ética y periodismo. Barcelona: Paidós.

Azurmendi, Ana: La reforma de RTVE. En: Azumermendi, Ana (dir). La reforma de la televisión pública española. Valencia: Tirant Lo Blanch, 269-334.

Bron, Christian M. (2010). Financing and supervision of public service broadcasting. European legislation and current national developments concerning fi- 
nancial and content-related supervision. En: Public Service Media: Money for Content, IRIS Plus, 2010-4.

Caro, Francisco (2006). Gestión de empresas informativas. Madrid: McGraw Hill.

Consejo para la reforma de los medios de comunicación de titularidad del estado (2005). Informe para la reforma de los medios de comunicación de titularidad del estado. Madrid.

European Commission (2009). 2009 Broadcasting Communication.

Fernández Alonso, Isabel y Santana, Fernanda (2000). Estado y medios de comunicación en la España democrática. Barcelona: Alianza

García Galera, María del Carmen (2006). Proteger la infancia. Los códigos de autorregulación a debate. En Icono 14 Revista de Comunicación y Nuevas Tecnologías, 7, 1-19.

Humphreys, Peter (1996). Mass Media and Media Policy in Western Europe. Manchester: Manchester University Press.

Manfredi Sánchez, Juan Luis (2004). Servicio público y calidad en la televisión pública. En Revista Andaluza de Administración Pública, 56, 275-297.

Manfredi Sánchez, Juan Luis (2009). Indicadores de RSC en la empresa periodística. En: Ámbitos: Revista internacional de comunicación, 18, 137-148

Murciano, Marcial (2006). Las políticas de comunicación y la construcción del Estado democrático. En Sala de Prensa, volumen 3. URL: http://www.saladeprensa.org/art670.htm: recuperado el 3 de septiembre de 2010.

Owen, Bruce M. y Wildman, Steven S. (1992). Video Economics. Boston: Harvard University Press

Pew Research Center (2010). Ideological News Sources: Who Watches And Why. Washington: Pew.

Picard, Robert G. (2001). Relations among Media Economics, Content, and Diversity. En: Nordicom Review, 20, 65-69.

Porter, Michael (2000). Estrategia competitiva: técnicas para el análisis de los sectores industriales y de la competencia. México DF: Compañía editorial continental.

Richeri, Giuseppe (1994). La transición de la televisión. Análisis del audiovisual como empresa de comunicación. Barcelona: Bosch.

Sánchez-Tabernero, Alfonso: El mercado televisivo europeo. La competencia entre las televisiones públicas y privadas. En: Telos: Cuadernos de comunicación e innovación, 63, 36-42. 
Sentencia del Tribunal de Justicia de las Comunidades Europeas (1974). Asunto C-155/73, Italia vs. G. Sacchi, 30 de abril.

Sentencia del Tribunal general de la Unión Europea de 1 de julio de 2010, Asuntos acumulados T-568/08 y T-573/08, Métropole télévision (M6) y Télévision française 1 SA (TF1) contra Comisión Europea

Varela, Juan (2008). RTVE.es tendrá publicidad como cualquier web comercial. En: Soitu.es [http://www.soitu.es/soitu/2008/05/13/sociedadcableada/1210676254_421988.html] recuperado el 29 de agosto de 2010.

\section{Referencia de este artículo}

Manfredi Sánchez, Juan Luis (2011). Escenarios y retos de la televisión pública en España. En: adComunica. Revista Científica de Estrategias, Tendencias e Innovación en Comunicación, $\mathrm{n}^{\mathrm{0}}$ 1. Castellón: Asociación para el Desarrollo de la Comunicación adComunica, Universidad Complutense de Madrid y Universitat Jaume I, 49-62. 